\title{
Deficits in temporal processing associated with autistic disorder
}

\author{
Melissa J. Allman* \\ Kennedy Krieger Institute, Johns Hopkins University School of Medicine, Baltimore, MD, USA \\ *Correspondence: allman@kennedykrieger.org
}

Currently idiopathic autism is typically diagnosed according to an observational assessment of certain behavioral, social, and cognitive tendencies across dimensions of language, social interaction, imaginative play, and restricted or repetitive cognitions and behavior. Parent interview is required to confirm presence of specific tendencies within the first 3 years of life. There is, as yet, no bio-medical test or marker for autism, although certain neurological, genetic, and physiological differences are known. There are often common co-morbid disorders, such as problems with sleep, motor function and attention-hyperactivity. An individual is diagnosed along spectrum of impairment (autistic disorder or autism spectrum disorder). Thus any psychological theory of autism is required to describe differences, and similarities, across a range of behavioral, social and cognitive abilities, within and between affected individuals. Arguably the three most dominant theories of autistic disorder relate to weak central coherence (or information processing), executive functioning, and theory of mind. There may be another hitherto, rather unacknowledged possibility: a deficit in temporal processing.

An adaptive sensitivity to the duration of events, and time between them, is critical to adaptive cognitive, behavioral, and social function. There are anecdotal and clinical reports of problems with time in autistic disorder, and successful applied behavioral educational and therapeutic supports with this population often include external signals to indicate the passage of time, temporal structure, and upcoming events. Currently, the empirical literature on temporal processing in autistic disorder is relatively scant, and encompasses findings spanning a range of psychology; neurological, genetic, behavioral, and cognitive, observational, and computational assessments. These include reports that children with autism experience difficulties imaging past and future changes of a current situation and understanding that successive events are part of a unitary process; differences in temporal reproduction and duration perception (in the interval range); and electrophysiological reports of disordered temporal binding of stimulus input, apparently over extended periods (than is typical). These findings are usually complementary, although vary greatly in the nature of the methods and analysis used (for a review, see Allman and Meck, 2011). There is preliminary evidence from data modeling to suggest that aspects of the perception of duration might be "developmentally delayed" in this population (see Allman et al., 2011).

Consistent among these aforementioned findings is the interpretation of results within the context of a deficit in (some aspect of) temporal processing in autistic disorder (e.g., Boucher et al., 2007). These range from suppositions that affected individuals experience problems thinking about time and higher temporal cognition, to notions that there are fundamental differences in the quality of the "subjective present" as it relates to the temporal processing of sensory information. Clock gene anomalies have been related to sleep, memory, and timing problems in autistic disorder. There have been several attempts to assert a hypothesis of temporal processing disturbance in autistic disorder, both within the discussion of published reports, or in more speculative accounts which go so far as to describe diagnostic features of autistic disorder as manifestations of atypical aspects of temporal processing (for details, see Allman and DeLeon, 2009). However, there is as yet, no well-defined profile of temporal processing abilities in these individuals, and no sufficiently specified theory.

Perhaps the appeal of a temporal deficit hypothesis of autism might be its ability to assimilate the traditional, and well supported accounts of autism into one, encompassing and testable hypothesis. It might also be possible to account for diagnostic features of the disorder. For instance, atypical social synchrony and temporal patterning may reduce the quality of social bonding, joint attention, and the to- and fro of social reciprocity, and communication (the latter are heavily implicated in autism). Differences in the ability to integrate sensory inputs and events over time may create problems with perception, learning and memory, and create problems of weak central coherence. Deficits in temporal processing might influence and be related to known differences in patterns of local and global information processing (couched within information-processing traditions), and executive functions (e.g., episodic memory, planning). Pathological restricted and repetitive behaviors and interests, rituals and routines, might otherwise have adaptive significance, such that they serve to parse or regulate temporal processing and compensate for a failure to predict events, and reduce posited disorientation in time. The relative length and complexity of rituals or routines has previously been posited to correspond to the extent (boundary) of temporal thought in affected individuals. Assuming that sensitivity to duration is related to our subjective sense of time, then autistic problems with imaginative play, empathizing, and theory of mind might be otherwise considered related to deficits in aspects of temporal processing - being able to export oneself into the mind of another person likely requires some form of "mental time travel."

It is important to stress that to-date the study of temporal processing in autism is in its infancy, however a temporal deficit hypothesis of autistic disorder does not appear premature given the relevant literature. At a more general level, there is little doubt that increased knowledge of temporal processing in autism is both necessary and likely fruitful both for the scientist, and the affected individual who has the potential to 
benefit from improved social and non-social temporal supports. Collectively, there are reasonable, and promising grounds to support a hypothesis of autism based on deficits in temporal processing.

\section{ACKNOWLEDGMENT}

The author is supported by a Pathway to Independence award (K99 HD058698) from the Eunice Kennedy Shriver National Institute of Child Health and Human Development.

\section{REFERENCES}

Allman, M. J., and DeLeon, I. G. (2009). "No time like the present: time perception in autism," in Causes and Risks for Autism, eds A. C. Giordano and V. A. Lombardi (New York: Nova Science Publishers), 65-76.

Allman, M. J., and Meck, W. H. (2011). Pathological distortions in time perception and timed performance. Brain (in press).

Allman, M. J., DeLeon, I. G., and Wearden, J. H. (2011). A psychophysical assessment of timing in individuals with autism. Am. J. Intellect. Dev. Disabil. 116, 165-178.

Boucher, J., Lind, S., and Williams, D. (2007). Temporal cognition in children with autistic spectrum disorder: tests of diachronic thinking. J. Autism Dev. Disord. 37, 1413-1429.

Received: 28 February 2011; accepted: 16 March 2011; published online: 25 March 2011.

Citation: Allman MJ (2011) Deficits in temporal processing associated with autistic disorder. Front. Integr. Neurosci. 5:2. doi: 10.3389/fnint.2011.00002

Copyright $(\odot 2011$ Allman. This is an open-access article subject to an exclusive license agreement between the authors and Frontiers Media SA, which permits unrestricted use, distribution, and reproduction in any medium, provided the original authors and source are credited. 